Originalien

Ophthalmologe 2021 $\cdot 118: 461-469$ https://doi.org/10.1007/s00347-021-01365-w Eingegangen: 11. Dezember 2020 Überarbeitet: 23. Februar 2021 Angenommen: 26. Februar 2021 Online publiziert: 29. März 2021 (c) Der/die Autor(en) 2021
Caroline Maria Glatzel ${ }^{1}$ Ágnes Patzkó ${ }^{2}$ Juliane Matlach ${ }^{3}$. Franz Grehn²

' Universitätsklinik für Dermatologie und Venerologie, Würzburg, Deutschland

${ }^{2}$ Universitäts-Augenklinik Würzburg, Würzburg, Deutschland

${ }^{3}$ Augenklinik, Universitätsmedizin Mainz, Mainz, Deutschland

\title{
Ergebnisse der filtrierenden Trabekulotomie (FTO) im Vergleich zur konventionellen Trabekulektomie (TE) - eine gematchte Fall-Kontroll-Studie
}

\section{Video online}

Die Online-Version dieses Beitrags (https:// doi.org/10.1007/s00347-021-01365-w) enthält ein Video zur Operationstechnik der filtrierenden Trabekulotomie. Beitrag und Video stehen Ihnen auf www. springermedizin.de zur Verfügung. Bitte geben Sie dort den Beitragstitel in die Suche ein, das Zusatzmaterial finden Sie beim Beitrag unter "Ergänzende Inhalte“.

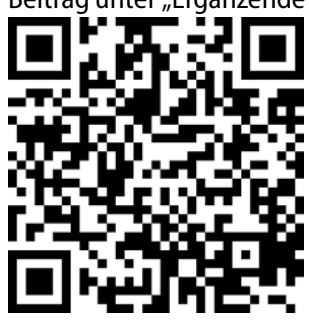

\section{Filtrierende Glaukomoperationen senken nach wie vor den Au- geninnendruck stärker als nicht penetrierende oder minimal inva- sive Glaukom (MIGS) Verfahren, erfordern aber eine aufwendige Nachbetreuung, um Misserfolge und Komplikationen zu vermeiden. In dieser Arbeit werden die 2-Jahres- Ergebnisse einer filtrierenden Trabe- kulotomie im gematchten Vergleich zur Trabekulektomie dargestellt. Dieses Verfahren ist gleich wirk- sam und stellt eine Alternative zur Trabekulektomie dar.}

Das Glaukom ist nach der Katarakt weltweit die zweithäufigste Erblindungsursache, jedoch die häufigste Ursache irreversibler Erblindung [33]. Nach einer Hochrechnung von Quigley et al. wird die beidseitige Erblindung durch Glaukom im Jahr 2020 weltweit auf 11,2 Mio. Menschen [22] und die Prävalenz des Glaukoms 2040 auf 111,2 Mio. Menschen [31] geschätzt. Aufgrund des demografischen Wandels ist der Anstieg der Glaukomprävalenz eine ernsthafte Herausforderung für die nähere Zukunft [5, 32].

Eine filtrierende Glaukomoperation senkt den intraokularen Druck (IOD) beim Glaukom sehr wirksam. Sie ist immer dann erforderlich, wenn sich Medikamente, Laser oder minimalinvasive Verfahren (MIGS) als unzureichend erwiesen haben $[16,19,20$, 30]. Seit 50 Jahren ist die Trabekulektomie eine Hauptstütze der primären Glaukomchirurgie [3, 15], aber ihre relativ hohe Komplikationsrate und der Nachbetreuungsaufwand geben immer wieder Anlass, Techniken mit gleicher Wirksamkeit, aber geringeren Nebenwirkungen anzustreben $[1,6,8,12-14$, 17, 23-25]. Die hier analysierte neue Operationstechnik „filtrierende Trabekulotomie“ (FTO) wurde mit diesem Ziel entwickelt. Das Prinzip der Operationsmethode basiert wie bei der Trabekulektomie auf subkonjunktivaler Filtration. Im Gegensatz zur Trabekulektomie ist der künstliche Abflussweg jedoch prinzipiell anders, da durch die seitliche Lage der Trabekulotomien die Vorderkammer an der Präparationsstelle geschlossen bleibt. Daher tritt kein schwallartiger Abfluss von Kammerwasser auf. Der Abfluss wird bereits durch die Ostien des Schlemm-Kanals und danach durch den Skleradeckel gedrosselt. Eine Iridektomie ist deshalb nicht erforderlich. Das chirurgische Konzept der FTO zielt auch auf eine diffusere Verteilung des Kammerwassers unter der Bindehaut.

Die vorliegende Studie analysiert die 2-Jahres-Ergebnisse dieser Operationsmethode. Die 1-Jahres-Ergebnisse wurden bereits zuvor publiziert [18].

\section{Material und Methode}

\section{Studiendesign}

Das Design dieser Fall-Kontroll-Studie wurde von der Ethikkommission der Medizinischen Fakultät der Universität Würzburg überprüft und positiv begutachtet (Nr. 298/14); 117 primäre Glaukomoperationen durch denselben Operateur (FG) wurden eingeschlossen, und zwar 30 konsekutive Patienten, bei denen eine filtrierende Trabekulotomie (FTO) ausgeführt wurde, und $87 \mathrm{~Pa}$ tienten als gematchte Kontrollgruppe, bei denen eine Trabekulektomie (TE) in den 4 Jahren davor erfolgt war. Um die Vergleichbarkeit zwischen FTOGruppe und Kontrollgruppe (TE) zu 


\begin{tabular}{|c|c|c|c|}
\hline Vor Operation & FTO & TE & Signifikanz $p$ \\
\hline Alter (Jahre) & $67,0 \pm 10,0$ & $66,9 \pm 9,2$ & 0,94 \\
\hline Geschlecht (m - \%) & 36,7 & 49,4 & 0,29 \\
\hline Augen (RA - \%) & 50,0 & 52,9 & 0,83 \\
\hline POWG (\%) & 66,7 & 77,0 & 0,33 \\
\hline PEX-Glaukom (\%) & 20,0 & 21,8 & 1,00 \\
\hline Pigmentglaukom (\%) & 13,3 & 1,1 & 0,02 \\
\hline Pseudophakie (\%) & 30,0 & 10,3 & 0,02 \\
\hline $\begin{array}{l}\text { Medikamente (\%) } \\
\text { (1/2/3/4 Wirkstoffklassen) }\end{array}$ & $\begin{array}{l}3,3 / 20,0 / 33,3 / \\
43,3\end{array}$ & $\begin{array}{l}11,5 / 25,3 / 37,9 / \\
24,1\end{array}$ & Jeweils $p>0,05$ \\
\hline IOD - alle Patienten $(\mathrm{mm} \mathrm{Hg})$ & 23,0 & 23,0 & 0,86 \\
\hline IOD nur PEX (mm Hg) & 21,0 & 25,0 & 0,32 \\
\hline $\begin{array}{l}\text { Visus Gesamtgruppe (logMAR (dezi- } \\
\text { mal)) }\end{array}$ & $0,05(0,9)$ & $0,10(0,8)$ & 0,56 \\
\hline Visus PEX-Gruppe (logMAR (dezimal)) & $0,19(0,63)$ & $0,00(1,0)$ & 0,39 \\
\hline
\end{tabular}

Tab. 2 Status 1,5 und 2 Jahre nach Operation (Matching 1:3 - FTO $n=27 ; \operatorname{TE} n=68$ )

\begin{tabular}{|c|c|c|c|}
\hline & FTO & TE & Signifikanz $p$ \\
\hline \multicolumn{4}{|l|}{ 1,5 Jahre postoperativ } \\
\hline Vollständiger Erfolg (\%) & 42,9 & 52,1 & 0,50 \\
\hline Qualifizierter Erfolg (\%) & 57,1 & 71,8 & 0,23 \\
\hline $\begin{array}{l}\text { Medikamente (\%) } \\
\text { (1/2/3/4 Wirkstoffklassen) }\end{array}$ & $0,0 / 17,9 / 10,7 / 3,6$ & $4,2 / 9,9 / 14,1 / 7,0$ & Jeweils $p>0,05$ \\
\hline IOD gesamt $(\mathrm{mm} \mathrm{Hg})$ & 13,4 & 12,4 & 0,18 \\
\hline IOD PEX (mm Hg) & $15,5(n=6)$ & $11,0(n=15)$ & 0,02 \\
\hline \multicolumn{4}{|l|}{2 Jahre postoperativ } \\
\hline Vollständiger Erfolg (\%) & 33,3 & 56,7 & 0,07 \\
\hline Qualifizierter Erfolg (\%) & 70,4 & 77,6 & 0,60 \\
\hline $\begin{array}{l}\text { Medikamente }(\%) \\
\text { (1/2/3/4 Wirkstoffklassen) }\end{array}$ & $\begin{array}{l}11,1 / 14,8 / \\
11,1 / 11,1\end{array}$ & $4,3 / 10,1 / 8,7 / 5,8$ & - \\
\hline IOD gesamt $(\mathrm{mm} \mathrm{Hg})$ & 12,8 & 11,0 & 0,12 \\
\hline IOD PEX $(\mathrm{mm} \mathrm{Hg})$ & $16,0(n=6)$ & $11,0(n=17)$ & 0,05 \\
\hline Visus Gesamtgruppe (logMAR (dezimal)) & $0,20(0,63)$ & $0,15(0,7)$ & $p>0,05$ \\
\hline Visus PEX-Gruppe (logMAR (dezimal)) & $0,05(0,9)$ & $0,13(0,7)$ & $p>0,05$ \\
\hline
\end{tabular}

erhöhen, wurden die Patienten beider Gruppen zuvor im Verhältnis 1:3 nach Alter und Augeninnendruck gematcht. Die Patienten waren aber auch in Bezug auf Sehschärfe, Geschlechtsverteilung, Verteilung der Glaukomformen POWG und PEX (TE-Gruppe 19,5\% PEXGlaukome; FTO-Gruppe 20,0\% PEXGlaukome) sowie bezüglich präoperativer Augenmedikation statistisch nicht signifikant unterschiedlich (• Tab. 1). Follow-up-Daten für die Zeitpunkte 1,5 und 2 Jahre nach der jeweiligen Glaukomoperation wurden entweder an der geschlossen. Patienten, die präoperativ systemische Medikamente zur Senkung des IOD erhielten, wurden nur eingeschlossen, wenn diese 3 oder mehr Tage vor der Operation abgesetzt wurden. Weitere Ausschlusskriterien waren ein primärer oder sekundärer Winkelverschluss, Normaldruckglaukom, angeborenes Glaukom, Neovaskularisationsglaukom (z. B. bei Diabetes mellitus oder nach Zentralvenenverschluss), absolutes Glaukom oder frühere inzisionelle Augenoperationen (z.B. kombinierte Phako-Trabekulektomie oder Netzhautoperationen) sowie Patienten mit mehr als 2 zykloablativen Eingriffen. Typ-2Diabetiker ohne okuläre Manifestationen waren kein Ausschlusskriterium.

\section{Endpunkte}

Als primärer Endpunkt wurde der 2-Jahres-Erfolg, definiert als ein IOD von $\leq 18 \mathrm{~mm} \mathrm{Hg}$ bei gleichzeitiger IOD-Reduktion um mindestens $30 \%$ im Vergleich zum präoperativen IOD, gewertet. Als „vollständiger Erfolg“ wurde gezählt, wenn diese IOD-Reduktion ohne Glaukommedikation erreicht wurde, als "qualifizierter Erfolg“, wenn für dieses Erfolgskriterium eine medikamentöse Zusatztherapie erforderlich war [29]. Als sekundäre Endpunkte wurden die mittlere Drucksenkung über 2 Jahre, die IOD-Entwicklung im Verlauf (für einzelne Patienten und im Gruppenvergleich), die Sehschärfe sowie Komplikationen bzw. Re-Operationen ausgewertet. Eine statistische Subgruppenanalyse erfolgte für Augeninnendruck und Sehschärfe der PEX-Glaukome, nicht aber für die Pigmentglaukome, deren Anteil hierfür zu klein war. Da evtl. Komplikationen meist früh nach der Operation auftraten, sind hier nur solche aus dem späteren Verlauf (>1 Jahr) berücksichtigt.

\section{Operationstechniken}

\section{Einschluss- und Ausschlusskriterien}

Es wurden Patienten mit primärem oder spezifischem sekundärem Offenwinkelglaukom (Pseudoexfoliationsglaukom [PEX] und Pigmentglaukom [PG]) ein-

\section{Filtrierende Trabekulotomie (FTO).}

Nach üblicher Präparation eines Fornixbasalen Bindehautlappens wurde Mitomycin C in 4 Schwämmchen $(2 \times 8 \mathrm{~mm}$; Flüssigkeitsaufnahme ca. $120 \mu \mathrm{l}$; Konzentration $0,2 \mathrm{mg} / \mathrm{ml} ; 3 \mathrm{~min}$ ) unter die Bindehaut und Tenon appliziert und da- 
Ophthalmologe 2021 · 118:461-469 https://doi.org/10.1007/s00347-021-01365-w

(c) Der/die Autor(en) 2021

\section{M. Glatzel · Á. Patzkó • J. Matlach • F. Grehn}

\section{Ergebnisse der filtrierenden Trabekulotomie (FTO) im Vergleich zur konventionellen Trabekulektomie (TE) - eine gematchte Fall-Kontroll-Studie}

\section{Zusammenfassung}

Ziel. Ziel dieser Studie war es, die 2-JahresErgebnisse der filtrierenden Trabekulotomie (FTO) im Vergleich zur konventionellen Trabekulektomie (TE) bei primärem Offenwinkelglaukom, Pseudoexfoliationsglaukom und Pigmentglaukom zu untersuchen.

Patienten und Methoden. Es wurden 30 konsekutive Patienten nach FTO und 87 Patienten nach TE nach intraokularem Druck (IOD) und Alter im Verhältnis 1:3 gematcht. Primärer Endpunkt war das Erreichen des Zieldrucks nach 2 Jahren. Als vollständiger Erfolg wurde ein IOD ohne Medikamente von $\leq 18 \mathrm{~mm} \mathrm{Hg}$ bei gleichzeitiger IOD-Reduktion um $\geq 30 \%$ definiert, als qualifizierter Erfolg, wenn hierfür zusätzlich Medikamente erforderlich waren. Sekundäre Endpunkte waren mittlere Drucksenkung, resultierende Sehschärfe, Komplikationen und nachfolgende Operatio- nen. Die Operationstechnik der filtrierenden Trabekulotomie ist als Video zu diesem Beitrag abrufbar.

Ergebnisse. Zwei-Jahres-Daten konnten von 27 Patienten aus der FTO-Gruppe und 68 Patienten aus der TE-Gruppe erhoben werden. Die Patienten beider Gruppen wurden vor Beginn der Studie bezüglich Alter und IOD gematcht, waren aber auch bezüglich Sehschärfe, Geschlecht und Medikation nicht unterschiedlich. Der Median des präoperativen IOD unter Therapie betrug in beiden Gruppen 23,0 mm Hg. Nach den oben genannten Kriterien wurde ein qualifizierter 2-Jahres-Erfolg bei 70,4\% der FTO-Gruppe und bei $77,6 \%$ der TE-Gruppe erzielt $(p=0,60)$, ein vollständiger 2-Jahres-Erfolg bei 33,3\% der FTO-Gruppe und bei $56,7 \%$ der TEGruppe $(p=0,07)$. Beide Operationsmethoden senkten den Augeninnendruck nach 24 Monaten signifikant $(p<0,001)$, und zwar auf $12,8 \mathrm{~mm} \mathrm{Hg}$ in der FTO-Gruppe und $11,0 \mathrm{~mm} \mathrm{Hg}$ in der TE-Gruppe. Die Sehschärfe war postoperativ bei beiden Gruppen etwas verringert, unterschied sich jedoch nicht signifikant zwischen beiden Gruppen. Komplikations- und Reoperationsrate waren gering und unterschieden sich nicht zwischen den Gruppen.

Schlussfolgerung. FTO und TE sind nach 2 Jahren weitgehend gleichwertig bezüglich Zieldruck, IOD-Senkung, Sehschärfe und Komplikationen.

Schlüsselwörter

Glaukomchirurgie - Filtrierende Glaukomchirurgie · Nicht-penetrierende Glaukomchirurgie . Augendrucksenkung $\cdot$ Matching

\section{Results of filtering trabeculotomy (FTO) compared to conventional trabeculectomy (TE)—a matched case control study}

\section{Abstract}

Objective. The aim of this study was to examine the 2-year results of filtering trabeculotomy (FTO) compared to conventional trabeculectomy (TE) in primary open-angle glaucoma, pseudoexfoliation glaucoma, and pigmentary glaucoma.

Patients and methods. Thirty consecutive patients after FTO and 87 patients after TE were included in the study. Both groups were matched 1:3 according to age and intraocular pressure (IOP). The primary endpoint was reaching the target IOP after 2 years. An IOP without medication of $\leq 18 \mathrm{~mm} \mathrm{Hg}$ and an IOP reduction of $\geq 30 \%$ were defined as complete success, and as qualified success with medication. Secondary endpoints were mean IOP reduction, resulting visual acuity, complications and subsequent operations. The surgical technique of the FTO is available as a video for this article.

Results. The 2-year data from 27 patients with FTO and 68 patients with TE were evaluated. The patients in both groups were matched according to age and IOP but were also homogeneous with respect to visual acuity, gender, and medication. The preoperative IOP with glaucoma medication was $23.0 \mathrm{~mm} \mathrm{Hg}$ in both groups. According to the defined criteria, a qualified 2-year success was achieved in $70.4 \%$ of the FTO group and in $77.6 \%$ of the TE group $(p=0.60)$ and a complete 2 -year success in $33.3 \%$ of the FTO group and $56.7 \%$ of the TE group $(p=0.07)$. The IOP was significantly reduced after 24 months in both surgical groups $(p<0.001)$ and was $12.8 \mathrm{~mm} \mathrm{Hg}$ in the FTO group and $11.0 \mathrm{~mm} \mathrm{Hg}$ in the TE group. Visual acuity was moderately reduced postoperatively but did not differ significantly between the two groups. Complication and reoperation rates were low and not different between both groups.

Conclusion. The results of FTO and TE are largely similar after 2 years in terms of complete and qualified success rate, lowering of IOP, visual acuity, and complications.

\section{Keywords}

Glaucoma surgery · Filtering glaucoma surgery - Non-penetrating glaucoma surgery . Lowering of intraocular pressure $\cdot$ Matching nach mit $30 \mathrm{ml}$ BSS gespült. Der äußere Skleradeckel wurde in halber Skleradicke in einer Größe von $4 \times 4 \mathrm{~mm}$ bis in die klare Hornhaut angelegt. Danach wurde ein zweiter, etwas kleinerer zungenförmiger tiefer Skleradeckel in einer Ebene direkt über dem Ziliarkörper präpariert, wodurch der Schlemm-Kanal lokalisiert und in gesamter Breite eröffnet werden konnte. Dabei wurde die Innenwand des Kanals erhalten und die Ostien des Schlemm-Kanals auf beiden Seiten freigelegt. Der tiefe Skleradeckel wurde abgetrennt. Die Ostien des Schlemm-Kanals wurden identifiziert und der SchlemmKanal mit Trabekulotomiesonden nach Mackensen (Geuder Ophthalmic Instruments, Heidelberg, Deutschland) kanüliert. Durch Einschwenken in die Vorderkammer mit Drehpunkt am De- ckelrand wird der Kanal auf beiden Seiten lediglich temporal bzw. nasal des Dissektionsbereichs eröffnet, während die freigelegte Innenwand des SchlemmKanals (Trabekel-Descemet-Membran) intakt bleibt. Daher ist eine Iridektomie nicht erforderlich. Abschließend wurde der äußere Skleradeckel mit 2 oder 4 einzelnen 10/0-Nylonfäden locker verschlossen. Wegen der Flussreduktion 


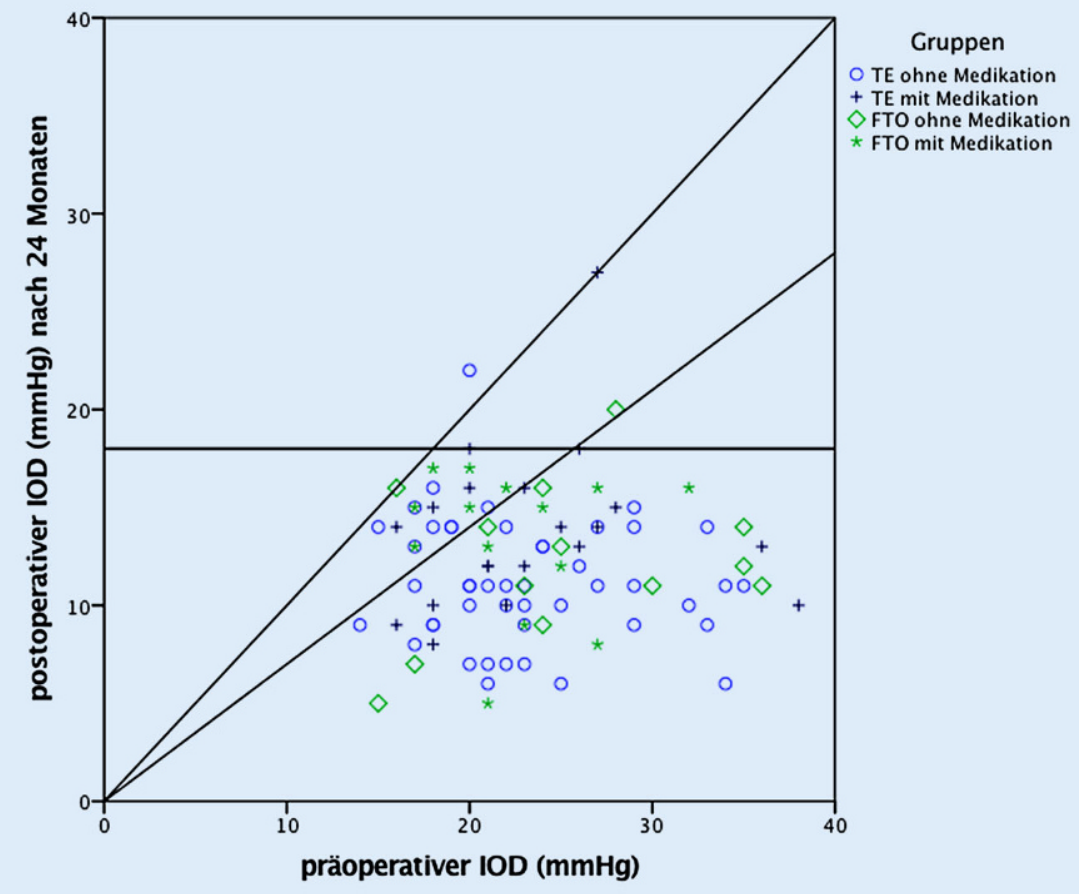

Abb. 1 ॥ Streudiagramm des Augeninnendrucks (IOD) vor und nach der Operation nach 24 Monaten. Datenpunkte oberhalb der 45-Grad-Linie zeigen eine postoperative Erhöhung des Augeninnendrucks an, Datenpunkte unterhalb der 45-Grad-Linie zeigen eine postoperative Senkung des Augeninnendrucks an. Datenpunkte unterhalb der horizontalen Linie von $18 \mathrm{~mm} \mathrm{Hg}$ und gleichzeitig unterhalb der Linie der 30 \%igen IOD-Senkung zeigen den vollständigen Erfolg (ohne Medikamente) bzw. qualifizierten Erfolg (mit Medikamenten). Kreise (o) und Rauten ( $\diamond$ ) sind 2-Jahres-Werte ohne Medikamente, plus (+) und Sterne $\left({ }^{*}\right)$ sind 2-Jahres-Werte mit Medikamenten. TE Trabekulektomie, FTO filtrierende Trabekulotomie

durch die Ostien des Schlemm-Kanals wurden diese Nähte lockerer als bei der konventionellen Trabekulektomie geknüpft. Die Bindehaut wurde durch eine fortlaufende 10/0-Nylon Matratzennaht [21] verschlossen. Die Operationstechnik ist als Video zu diesem Beitrag abrufbar.

Trabekulektomie (TE). Bei der TE wurde der Skleradeckel mit $4 \times 3,5 \mathrm{~mm}$ etwas kleiner dimensioniert. Mitomycin C wurde, wie oben beschrieben, angewendet. Zum Öffnen der Vorderkammer wurde ein $0,8 \times 2$ mm großes Fenster mit $2 / 3$ Fläche in der klaren Hornhaut ausgeschnitten (Trabekulektomie) und eine periphere Iridektomie angelegt. Der Skleradeckel wurde mit 2 oder 4 10/0-Nylonnähten verschlossen, deren Spannung je nach Abflussleichtigkeit (BSS-Injektion in die Vorderkammer) justiert wurde.

\section{Statistische Methoden}

Die statistische Analyse wurde mit SPSS (Version 23, IBM, Ehningen, Deutschland) und SAS Macro (Version 9.3, IBM, Ehningen, Deutschland) durchgeführt. $p$-Werte von $\leq 0,05$ wurden als statistisch signifikant angesehen. Die 117 rekrutierten Patienten wurden auf der Grundlage ihres Alters (Bereich \pm 8 Jahre) und ihres präoperativen IOD (Bereich $\pm 3 \mathrm{~mm} \mathrm{Hg}$ ) im Verhältnis 1:3 in eine Fallgruppe und eine Kontrollgruppe eingeteilt. Die Fallgruppe (FTO) bestand zu Beginn der Studie aus 30 Patienten, von denen 27 Patienten in der vorliegenden Studie 2 Jahre lang beobachtet werden konnten. $\mathrm{Zu}$ Beginn der Studie bestand die Kontrollgruppe (TE) aus 87 Patienten, von denen nach 2 Jahren Daten von 68 Patienten erhoben werden konnten. Dichotome Variablen wurden mit dem Fisher-Exact-Test auf Signifikanz geprüft, kontinuierliche Variablen wie
Augeninnendruck oder Sehschärfe wurden mit dem Shapiro-Wilk-Test getestet. Ungepaarte, nicht normalverteilte Stichproben (z. B. Variabilitäten unter Patienten) wurden unter Verwendung des Mann-Whitney-U-Tests und ungepaarte normalverteilte Proben mit dem ungepaarten Students' t-Test getestet. Nicht normalverteilte Variablen unter gepaarten Proben (IOD-Entwicklung eines Patienten) wurden mit dem WilcoxonVorzeichen-Rang-Test analysiert. Wenn beide Variablen normal verteilt waren, wurde der gepaarte Students' t-Test verwendet. Zur Analyse der Dauer des postoperativen Erfolgs (Überlebenszeit der beiden jeweiligen Operationen bezüglich vollständigen oder qualifizierten Erfolgs) wurde die kumulative KaplanMeier-Kurve berechnet. Zur besseren Anschaulichkeit wurden die Daten entsprechend den Guidelines der World Glaucoma Association auch in einem Streudiagramm (intraindividueller Vergleich) und als Boxplots (Augeninnendruckverlauf) dargestellt [29]. BoxplotDiagramme zeigen den Median sowie die $25 \%$ - und die $75 \%$-Perzentile, T-Balken die $10 \%$ - und $90 \%$-Perzentilen an. Daten außerhalb dieser Perzentilen wurden als zusätzliche Punkte in der Grafik angezeigt. Für normalverteilte Stichproben wurde ein Mittelwert mit Standardabweichung berechnet, andernfalls wurden der Median und der Interquartilbereich (IQR) verwendet.

\section{Ergebnisse}

Vergleichbarkeit der Studiengruppen.

Der Median des präoperativen Augeninnendrucks beider Studiengruppen war gleich und betrug $23,0 \mathrm{~mm} \mathrm{Hg}$ (IQR 20,00-27,00) [18], da die Fälle gematcht waren. Die durchschnittliche Zahl der präoperativ gegebenen lokalen Glaukommedikamente war in beiden Gruppen nicht signifikant unterschiedlich (•Tab. 1).

Nach 2 Jahren konnten 27 Patienten der FTO-Gruppe und 68 Patienten der TE-Gruppe nachuntersucht werden. Die Patienten beider Gruppen waren hinsichtlich Alter $\left(p_{\text {präop }}=0,94\right)$ und Geschlecht $\left(p_{\text {präop }}=0,29\right)$ vergleichbar. Vor der Operation umfasste die FTO-Grup- 
pe signifikant mehr pseudophake Augen als die TE-Gruppe $(p=0,02)$, nach 2 Jahren war der Unterschied nicht mehr signifikant $(p=0,15)$. Zirka drei Viertel aller Patienten hatten ein primäres Offenwinkelglaukom $\left(\mathrm{POWG}_{\text {präop }}=74,4 \%\right.$, $\left.\mathrm{POWG}_{2 \mathrm{a}}=70,5 \%\right)$, ca. ein Fünftel ein PEX-Glaukom $\left(\mathrm{PEX}_{\text {präop }}=21,4 \%, \mathrm{PEX}_{2 \mathrm{a}}=\right.$ $24,2 \%)$ und ca. $1 / 20$ ein Pigmentglaukom $\left(\mathrm{PG}_{\text {präop }}=4,3 \%, \mathrm{PG}_{2 \mathrm{a}}=5,3 \%\right)$. Die Verteilung des PEX-Glaukoms war in der FTO- bzw. TE-Gruppe innerhalb statistischer Grenzen gleich (• Tab. 1). Die Zahl der Patienten mit Pigmentdispersionsglaukom war hingegen in der FTO-Gruppe signifikant höher als in der TE-Gruppe $\left(P_{\text {präop }}=0,02, p_{2 a}=0,02\right)$. Die Zahl der pseudophaken Augen war präoperativ in der FTO-Gruppe höher, nach 2 Jahren jedoch nicht mehr signifikant unterschiedlich (•Tab. 2), d.h. der Zuwachs an Kataraktoperationen war nach TE höher als nach FTO.

\section{Erfolgsrate der Augendrucksenkung.}

Ein Jahr nach der Operation wurde ein vollständiger Erfolg in beiden Operationsgruppen bei ca. $80 \%$ der Patienten $(\mathrm{FTO}=79,3 \% ; \mathrm{TE}=83,1 \%)$ gefunden [18]. Nach 1,5 Jahren betrug der vollständige Erfolg $42,9 \%$ in der FTO- und $52,1 \%$ in der TE-Gruppe und nach 2 Jahren 33,3\% in der FTO- und 56,7\% in der TE-Gruppe. Der qualifizierte Erfolg betrug nach 1 Jahr $86,2 \%$ in der FTO- und $83,1 \%$ in der TE-Gruppe [18]. Nach 1,5 Jahren sank der qualifizierte Erfolg in beiden Gruppen ab $(\mathrm{FTO}=57,1 \%$; $\mathrm{TE}=71,8 \%)$ und betrug 2 Jahre nach der Operation $70,4 \%$ in der FTO-Gruppe und 77,6\% in der TEGruppe. Zwischen den Gruppen unterschieden sich vollständiger und qualifizierter Erfolg zu keinem Zeitpunkt signifikant (vollständiger Erfolg: $p_{12 \mathrm{Mon}}=0,78$; $p_{18 \mathrm{Mon}}=0,50 ; p_{24 \mathrm{Mon}}=0,07$. Qualifizierter Erfolg: $p_{12 \mathrm{Mon}}=1,00 ; p_{18 \mathrm{Mon}}=0,23$; $\left.p_{24 \mathrm{Mon}}=0,60\right)$, d.h. die Abnahme der Druckregulation beider Verfahren, war während der gesamten Beobachtungszeit weitgehend gleich (• Tab. 2). Die 2-Jahres-Ergebnisse des vollständigen und des qualifizierten Erfolgs aller Patienten sind im Streudiagramm von • Abb. 1 dargestellt und lassen die Drucksenkung jedes einzelnen Auges nachvollziehen. Die
Hier steht eine Anzeige.

글 Springer 

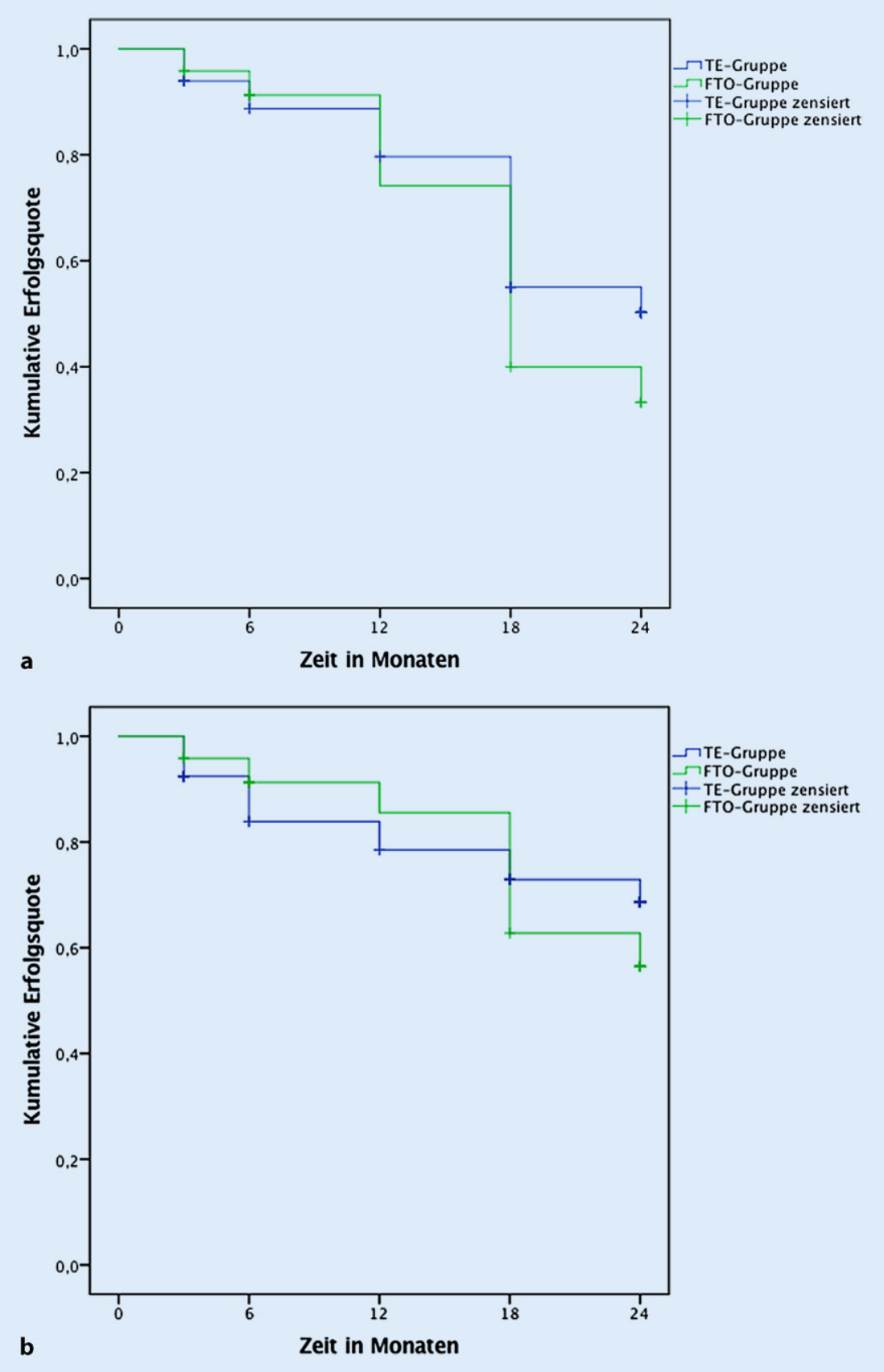

Abb. 2 \& a Kaplan-Meier-Überlebenskurve mit vollständigem Erfolg nach Trabekulektomie (TE) und filtrierender Trabekulotomie (FTO). Die Definition des vollständigen Erfolgs war ein Augeninnendruck (IOD) $\leq 18 \mathrm{~mm} \mathrm{Hg}$ und eine IOD-Reduktion von $\geq 30 \%$ ohne Medikamente. Präoperative Daten und $\leq 12$-Monats-Daten wurden nach Matlach et al. eingefügt [18]. b Kaplan-Meier-Überlebenskurve für qualifizierten Erfolg nach Trabekulektomie (TE) und filtrierender Trabekulotomie (FTO). Die Definition des qualifizierten Erfolgs war ein Augeninnendruck (IOD) $\leq 18 \mathrm{~mm} \mathrm{Hg}$ und eine IOD-Reduktion von $\geq 30 \%$ mit Medikamenten. Präoperative Daten und $\leq 12$-Monats-Daten wurden nach Matlach et al. eingefügt [18] postoperative Medikation ist in • Tab. 2 aufgeführt.

Kaplan-Meier-Analyse. Im ersten Jahr war der vollständige Erfolg in der FTOGruppe höher als in der TE-Gruppe, im zweiten Jahr war dagegen der vollständige Erfolg in der TE-Gruppe höher. Basierend auf der Kaplan-Meier-Überlebenskurve, betrug die Wahrscheinlichkeit eines vollständigen Erfolgs nach 1,5 Jahren in der TE-Gruppe 55,0 \% und in der FTOGruppe 39,9\%, nach 2 Jahren in der TEGruppe 50,2\% und in der FTO-Gruppe $33,3 \%$ (-Abb. 2a). Ein qualifizierter Erfolg wurde in der Kaplan-Meier-Überlebenskurve nach 1,5 Jahren in $72,9 \%$ (TE) und 62,7\% (FTO), nach 2 Jahren in $68,6 \%$ (TE) und 56,5\% (FTO) erreicht (- Abb. 2b).

\section{Verlauf des Augeninnendrucks nach} 1,5 bzw. 2 Jahren. Nach 1,5 Jahren betrug der IOD (ggf. unter zusätzlicher Therapie) in der FTO-Gruppe $13,4 \pm 4,2 \mathrm{mmHg}$, in der TE-Gruppe $12,4 \pm 3,2 \mathrm{mmHg}$, nach 2 Jahren in der FTO-Gruppe $12,8 \pm 3,8 \mathrm{~mm} \mathrm{Hg}$ und in der TE-Gruppe $11,0 \mathrm{~mm} \mathrm{Hg}$ (IQR 9,0-13,0). Nach 1,5 bzw. 2 Jahren unterschied sich der IOD zwischen diesen Gruppen nicht signifikant $\left(p_{18 \mathrm{Mon}}=0,18\right.$, $\left.p_{24 \mathrm{Mon}}=0,12\right)(\bullet$ Abb. 3$)$.

Ohne Berücksichtigung einer prozentualen Drucksenkung, d.h. bei starrer Obergrenze (vgl. Methode), war der 2-Jahres-Augeninnendruck ( \pm Medikamente) bei $97,0 \%$ der TE-Gruppe und bei $100 \%$ der FTO-Gruppe unter $21 \mathrm{~mm} \mathrm{Hg}$, bei $94,1 \%$ der TE-Patienten und bei 96,3\% der FTO-Patienten unter $18 \mathrm{~mm} \mathrm{Hg}$, und bei 80,9\% der TE-Patienten und bei 59,3\% FTO-Patienten unter $15 \mathrm{~mm} \mathrm{Hg}$ (vgl. - Abb. 1). Alle postoperativen intraokularen Druckwerte waren im Vergleich zum präoperativen IOD signifikant erniedrigt (• Abb. 3) sowohl in den gepoolten Werten aller Operationen als auch in den beiden Gruppen (FTO und TE) getrennt.

Sehschärfe. Die korrigierte Sehschärfe für die Ferne (SF) betrug vor Operation in der TE-Gruppe 0,10logMAR (dezimal 0,8) und in der FTO-Gruppe $0,05 \log$ MAR (dezimal 0,$9 ; p=0,6$ ) 


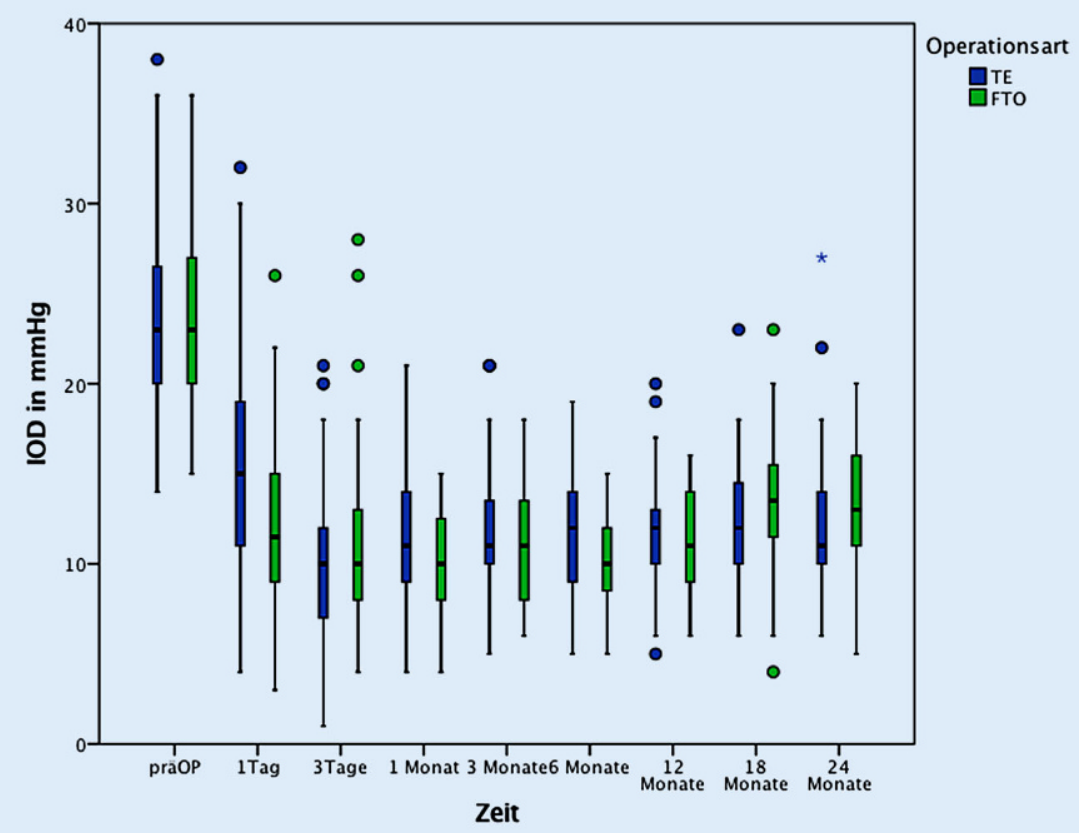

Abb. 3 ム Prä- und postoperativer Augeninnendruck (IOD) von Trabekulektomie (TE) und filtrierender Trabekulotomie (FTO). Der IOD (Median \pm IQR) unterscheidet sich nach 1,5 Jahren und 2 Jahren nicht signifikant zwischen den beiden chirurgischen Eingriffen. Punkte sind moderate, Sterne sind extreme Ausreißer. Präoperative Daten und $\leq 12$-Monats-Daten wurden nach Matlach et al. eingefügt [18]

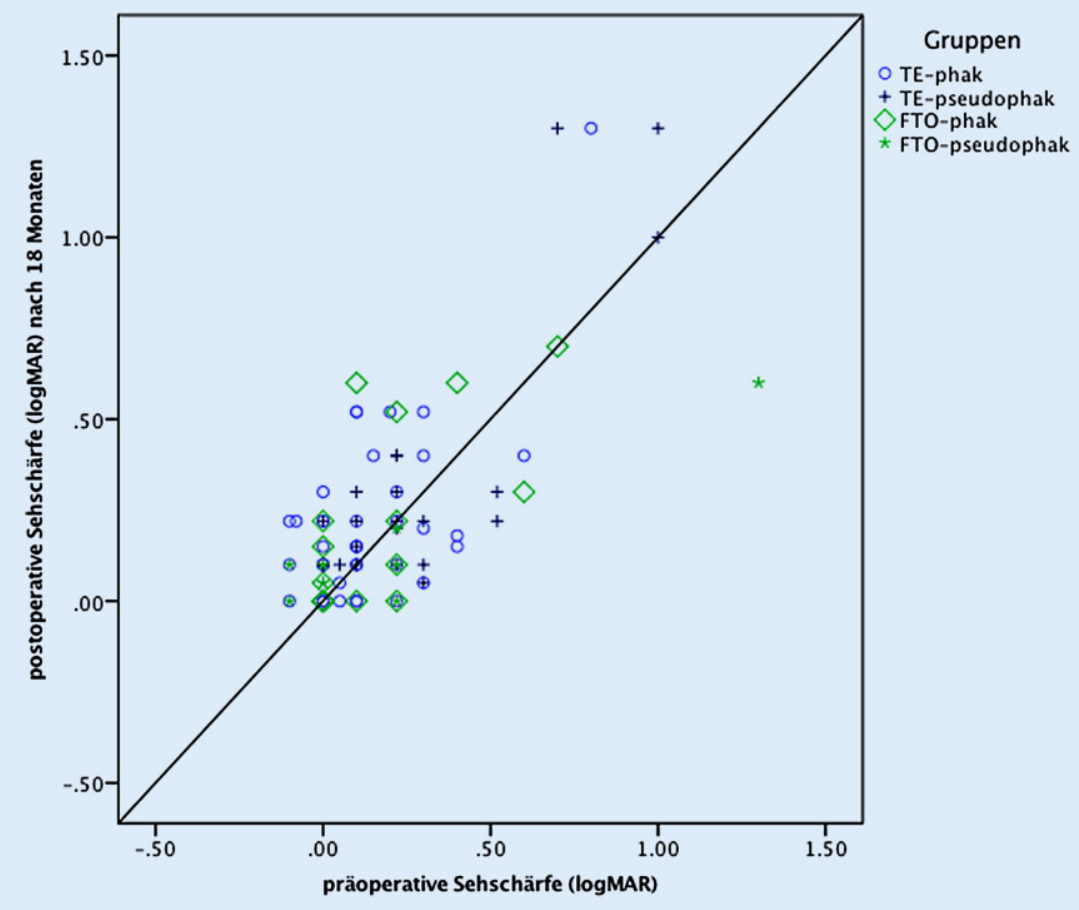

Abb. 4 \ Prä- und postoperative Sehschärfe nach 2 Jahren. Die präoperative Sehschärfe (logMAR) ist auf der $x$-Achse aufgetragen, die postoperative Sehschärfe auf der y-Achse. Werte oberhalb der 45Grad-Linie stellen einen Verschlechterung der Sehschärfe dar, Werte unterhalb der Linie eine postoperative Verbesserung der Sehschärfe.
[18] und 2 Jahre postoperativ bei TE $0,15 \log$ MAR (dezimal 0,7 ) und bei FTO $0,20 \log$ MAR (dezimal 0,63). Die postoperative SF war in beiden Gruppen im Vergleich zum präoperativen SF-Wert signifikant reduziert $(p<0,05)$. Die SF unterschied sich jedoch zwischen beiden Operationstechniken nicht signifikant $(p \geq 0,5)$ (• Tab. 2). Das Streudiagramm von $\triangle$ Abb. 4 zeigt den individuellen Sehschärfenvergleich aller Augen vor Operation und nach 2 Jahren.

Pseudoexfoliationsglaukom (Subgruppenanalyse) und Pseudophakie. Der postoperative Augeninnendruck (mit und ohne Medikamente) von Patienten mit PEX-Glaukom war nach Operation signifikant niedriger als präoperativ $(p<0,001)$. Die Unterschiede zwischen FTO und TE waren zwar grenzwertig signifikant, die Zahl der FTO Operationen jedoch sehr klein (•Tab. 2). Die postoperative Sehschärfe war während des gesamten Follow-up sowohl bei phaken als auch bei pseudophaken PEX-Augen nicht signifikant verringert (• Tab. 2). Der Zuwachs an Kataraktoperationen innerhalb der 2 Jahre war nach TE höher (14,0\%-Punkte) als nach FTO (9,9\%Punkte) (• Tab. 1 und 2). Die Anzahl der Patienten mit Pigmentglaukom war zu gering, um eine separate Bewertung zu ermöglichen (• Tab. 2).

Postoperative Komplikationen. Mögliche Komplikationen wie langfristige Hypotonie mit flacher Vorderkammer, hypotensive Makulopathie, Aderhautschwellung sowie externe Sickerkissenfistel, Blebitis oder Endophthalmitis wurden bei beiden Operationsmethoden nach 1,5 und 2 Jahren nicht beobachtet. Kein Patient beider Gruppen zeigte nach 1,5 bzw. 2 Jahren einen Augeninnendruck von $\leq 5 \mathrm{~mm} \mathrm{Hg}$.

Reoperationen. In der FTO-Gruppe wurden ein Sickerkissen-Needling (nach 1,5 Jahren) und eine Suturolyse (nach 2 Jahren) sowie eine YAG-Laser-Goniopunktur durchgeführt. In der TEGruppe wurden bei 2 Augen innerhalb von 2 Jahren eine ALT und bei 1 Auge ein Sickerkissen-Needling durchgeführt (- Tab. 2). 


\section{Diskussion}

Seit ihrer Erstbeschreibung durch Cairns im Jahr 1968 [3] erwies sich die Trabekulektomie über 50 Jahre lang als die effektivste augendrucksenkende Operation. Allerdings sind Nachbehandlung und Beherrschung von Komplikationen anspruchsvoll. Durch die Entwicklung der nicht penetrierenden Glaukomchirurgie [23, 24], Verwendung moderner Schlauchimplantate [4] und Einführung minimal-invasiver Glaukomeingriffe (MIGS) [2, 11, 26-28] hat sich das operative Spektrum in den letzten 10 Jahren stark erweitert, sodass jetzt die Operationswahl stärker auf die individuelle Situation des Patienten fokussiert werden kann. Nach wie vor sind aber die sog. Filtrationseingriffe, also die Ableitung des Kammerwassers unter die Bindehaut ohne Schlauchsysteme bei Primäreingriffen stärker und dauerhafter drucksenkend als alle anderen genannten Verfahren $[9,10,24]$.

Die vorliegende Studie vergleicht eine neue Operationsmethode, die filtrierende Trabekulotomie (FTO) mit der Trabekulektomie (TE) unter der Zielsetzung, das Nebenwirkungsspektrum zu verbessern, ohne den drucksenkenden Effekt des Eingriffs zu reduzieren. Die hier vorgelegten 1,5- und 2-Jahres-Ergebnisse ergänzen die bereits publizierten 1-JahresDaten [18].

Im Gegensatz zu den meisten anderen Studien, die als Erfolgskriterium Grenzwerte von 21 oder $18 \mathrm{~mm} \mathrm{Hg}$ festlegen, wurde entsprechend den Vorschlägen der WGA und EGS [7, 29] eine zusätzliche prozentuale Augendrucksenkung (hier $30 \%$ ) zugrunde gelegt, was immer dann von Bedeutung ist, wenn die Augeninnendruckwerte bereits vor der Operation nicht sehr stark erhöht sind. Andernfalls würden auch Operationen als Erfolg klassifiziert, die individuell keine oder nur eine geringe Augendrucksenkung erreicht haben. Das Streudiagramm von - Abb. 1 erlaubt es aber, auch andere Zieldruckkriterien für die hier vorgestellten Daten anzuwenden.

Das Prinzip der Operationsmethode „filtrierende Trabekulotomie“ (FTO) besteht darin, die Vorderkammer an der Operationsstelle nicht zu eröffnen (anders als bei der Trabekulektomie), sondern wie bei der tiefen Sklerektomie [25] eine Descemet-Trabekel-Membran im Operationsgebiet zu erhalten, sodass kein Irisprolaps erfolgt und keine Iridektomie erforderlich wird. Die Filtration wird erreicht, indem der benachbarte Schlemm-Kanal nach beiden Seiten mit einer Sondentrabekulotomie eröffnet wird, sodass Kammerwasser über die Ostien des Schlemm-Kanals in den Sklerasee unter den Skleradeckel fließen kann und danach unter die Bindehaut gelangt, wo es ähnlich der TE resorbiert wird (s. Video). Da bei dieser Methode bewusst eine Filtration angestrebt wird, erfolgt wie bei der TE eine intraoperative Applikation von MMC. Der Kammerwasserfluss wird bei der FTO zunächst durch Ostien des Schlemm-Kanals reguliert, die das Kammerwasser in den Sklerasee leiten. Der zweite Widerstand wird durch den Skleradeckel erzeugt, von wo das Kammerwasser in den subkonjunktivalen Raum gelangt. Im Gegensatz dazu wird bei TE der Kammerwasserfluss allein durch einen einzigen Widerstand, nämlich am Skleradeckel kontrolliert, hauptsächlich durch die Anzahl und Spannung der Nähte. Durch die 2-stufige Kontrolle des Abflusses bei der FTO sind die Sickerkissen diffuser verteilt und weniger dünn-zystisch als bei TE. Während 1 Jahr nach Operation beide Methoden eine gleiche Drucksenkung erbringen [18], ist nach 2 Jahren, gemessen am „vollständigen Erfolg“, die FTO etwas weniger wirksam als die TE, wenn auch nicht statistisch signifikant (- Abb. 2a und 3; - Tab. 2).

Insgesamt wurden im Beobachtungszeitraum von 2 Jahren keine signifikanten Unterschiede in der Drucksenkung beider Methoden gefunden. Betrachtet man jedoch den Gesamtverlauf aller Untersuchungszeitpunkte im 1. und 2. Jahr, dann lässt sich im 2. Jahr eine (nicht signifikante) Tendenz zu besserer Drucksenkung nach Trabekulektomie (TE) erkennen (• Abb. 3). Andererseits ist dieser neue Filtrationseingriff (FTO) wahrscheinlich stärker wirksam als nicht-penetrierende Verfahren (tiefe Sklerektomie, Kanaloplastik), wenn man die randomisierten Vergleichsstudien nicht-penetrierender
Verfahren gegenüber der Trabekulektomie zugrunde legt $[17,23,24]$.

Auch bezüglich Visuserhalt wurden in unserer Studie keine signifikanten Unterschiede zur Trabekulektomie gefunden, auch nach Berücksichtigung der unterschiedlichen Zahl pseudophaker Augen in beiden Gruppen. Die Subgruppenanalyse der Augen mit Pseudoexfoliation zeigte trotz höherer Ausgangsdruckwerte eine gleich effektive Augeninnendrucksenkung, und auch im Visusverlauf konnte kein Unterschied zu Augen mit primärem Offenwinkelglaukom gefunden werden (•Tab. 2). Bei keinem der Patienten traten schwerwiegende Komplikationen auf, weder in der frühpostoperativen Phase [18] noch langfristig. Nach 2 Jahren hatte die FTO die gleiche Komplikationsrate wie die TE (• Tab.2).

Durch das Matchingverfahren im Verhältnis 1:3 wird ein möglicher Bias zwischen beiden Gruppen stärker reduziert als bei 1:1-Matching*. Die Schwere des Glaukomschadens wurde nicht gesondert ins Studienprotokoll aufgenommen, da der ursprüngliche Ansatz, die Gesichtsfeldprogression zu ermitteln, über einen Zeitraum von 1 bis 2 Jahren nicht aussichtsreich erschien. Ein Bias durch unterschiedliche Glaukomvorschädigung beider Gruppen erscheint jedoch wenig wahrscheinlich, da beide Operationsverfahren unter gleicher Indikationsstellung des Operateurs durchgeführt wurden (FTO ausschließlich anstelle von TE). Eine multivariate Analyse von verschiedenen Systemerkrankungen (z.B. Diabetes mellitus) wurde wegen der begrenzten Fallzahl nicht durchgeführt, insbesondere neovaskuläre und entzündliche Glaukome waren aber laut Studienprotokoll ausgeschlossen.

Trotz der Einschränkung durch den zwar gematchten, jedoch nicht randomisierten Vergleich und die limitierte Fallzahl zeigt diese Studie eine gute Wirksamkeit dieses neuen Filtrationseingriffes.

\section{Fazit}

Die filtrierende Trabekulotomie (FTO) entspricht in Bezug auf Augeninnendruck, Sehschärfe und Erfolgsrate der Trabekulektomie (TE). Nach 2 Jahren 
war die Komplikationsrate in beiden Gruppen sehr gering und nicht signifikant unterschiedlich. Die FTO erwies sich als eine gute und sichere Alternative zur TE. Die vorliegende Studie rechtfertigt eine Weiterentwicklung dieser neuen Operationstechnik.

\section{Korrespondenzadresse

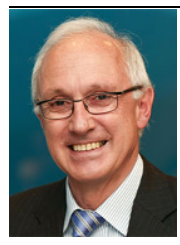 \\ Prof. Dr. Dr. h.c. Franz Grehn Universitäts-Augenklinik Würzburg Josef Schneider Str. 11, 97080 Würzburg, \\ Deutschland grehn_f@ukw.de}

Danksagung. Wir danken Frau Viktoria Rücker, Lehrstuhl für Klinische Epidemiologie und Biometrie, Universitätsklinikum Würzburg, Josef Schneider Str. 2, 97080 Würzburg, für die Beratung bei der statistischen Auswertung der Daten.

Funding. Open Access funding enabled and organized by Projekt DEAL.

\section{Einhaltung ethischer Richtlinien}

Interessenkonflikt. J. Matlach:DFG, Novartis - Forschungsförderung (nicht im Zusammenhang mit diesem Manuskript). F. Grehn: SANTEN Inc. - Advisoryboard-Mitglied (bis 2019). C.M. Glatzel und Á. Patzkó geben an, dass kein Interessenkonflikt besteht.

Alle beschriebenen Untersuchungen am Menschen oder an menschlichem Gewebe wurden mit Zustimmung der zuständigen Ethikkommission, im Einklang mit nationalem Recht sowie gemäß der Deklaration von Helsinki von 1975 (in der aktuellen, überarbeiteten Fassung) durchgeführt. Von allen beteiligten Patienten liegt eine Einverständniserklärung vor.

Open Access. Dieser Artikel wird unter der Creative Commons Namensnennung 4.0 International Lizenz veröffentlicht, welche die Nutzung, Vervielfältigung, Bearbeitung, Verbreitung und Wiedergabe in jeglichem Medium und Format erlaubt, sofern Sie den/die ursprünglichen Autor(en) und die Quelle ordnungsgemäß nennen, einen Link zur Creative Commons Lizenz beifügen und angeben, ob Änderungen vorgenommen wurden.

Die in diesem Artikel enthaltenen Bilder und sonstiges Drittmaterial unterliegen ebenfalls der genannten Creative Commons Lizenz, sofern sich aus der Abbildungslegende nichts anderes ergibt. Sofern das betreffende Material nicht unter der genannten Creative Commons Lizenz steht und die betreffende Handlung nicht nach gesetzlichen Vorschriften erlaubt ist, ist für die oben aufgeführten Weiterverwendungen des Materials die Einwilligung des jeweiligen Rechteinhabers einzuholen.

Weitere Details zur Lizenz entnehmen Sie bitte der Lizenzinformation auf http://creativecommons.org/ licenses/by/4.0/deed.de.

\section{Literatur}

1. Ang GS, Fenwick EK, Constantinou M et al (2020) Selective laser trabeculoplasty versus topical medication as initial glaucoma treatment: the glaucoma initial treatment study randomised clinical trial. Br JOphthalmol 104:813-821

2. Buffault J, Baudouin C, Labbé A (2019) XEN $\left({ }^{\circledR}\right)$ Gel Stent for management of chronic open angle glaucoma: a review of the literature. J Fr Ophtalmol 42:e37-e46 (Review)

3. Cairns JE (1968) Trabeculectomy, perliminary report of a new method. Am J Ophthalmol 66:673-679

4. Christakis PG, Zhang D, Budenz DL, Barton K, Tsai JC, Ahmed IIK (2017) Five-year pooled data analysis of the Ahmed Baerveldt comparison study and the Ahmed versus Baerveldt study. Am J Ophthalmol 176:118-126

5. Dietlein TS, Hermann MM, Jordan JF (2009) The medical and surgical treatment of glaucoma. Dtsch Arztebl Int 106:597-605

6. Do AT, Parikh H, Panarelli JF (2020) Subconjunctival microinvasive glaucoma surgeries: an update on the Xen gel stent and the PreserFlo MicroShunt. Curr Opin Ophthalmol 31:132-138

7. European GlaucomaSociety (2020)European Glaucoma Society terminology and guidelines for glaucoma, 5th edition. PubliComm, Savona. https:// www.eugs.org/eng/guidelines.asp, Zugegriffen: 10Dez 2020

8. Francis BA, Akil H, Bert BB (2017) Ab interno Schlemm's canal surgery. Dev Ophthalmol 59:127-146

9. Gedde SJ, Feuer WJ, Lim KS, Barton K, Goyal S, Ahmed IIK, Brandt JD (2020) Treatment outcomes in the primary tube versus trabeculectomy study after 3 years of follow-up. Ophthalmology 127:333-345

10. Gedde SJ, Feuer WJ, ShiW, Lim KS, Barton K, GoyalS Ahmed IIK, Brandt J (2018) Treatment outcomes in the primary tube versus trabeculectomy study after 1 year of follow-up.primary tube versus trabeculectomy study group. Ophthalmology 125:650-663

11. Hengerer FH, Kohnen T, Mueller M, ConradHengerer I (2017) Ab Interno gel implant for the treatment of glaucoma patients with or without prior glaucoma surgery: 1-year results. J Glaucoma 26:1130-1136

12. Jünemann AGM, Rejdak R, Hohberger B (2018) Trabecular micro-invasive glaucoma surgery: procedures and clinical results. Ophthalmologe 115:363-369

13. Khaw PT, Chiang M, Shah P, Sii F, Lockwood A, Khalili A (2017) Enhanced trabeculectomy: the Moorfields safer surgery system. Dev Ophthalmol 59:15-35

14. Klink T, Matlach J, Grehn F (2012) Non-penetrating glaucoma surgery. Ophthalmologe 109:807-815

15. Landers J, Martin K, Sarkies N, Bourne R, Watson P (2012) A twenty-year follow-up study of trabeculectomy: risk factors and outcomes. Ophthalmology 119:694-702

16. Lichter PR, Musch DC, Gillespie BW, Guire KE, Janz NK, Wren PA, Mills RP (2001) Interim clinical outcomes in the collaborative initial glaucoma treatment study comparing initial treatment randomized to medications or surgery. Ophthalmology 108:1943-1953

17. Matlach J, Dhillon C, Hain J, Schlunck G, Grehn F, Klink T (2015) Trabeculectomy versus canaloplasty (TVC study) in the treatment of patients with open-angle glaucoma: a prospective randomized clinical trial. Acta Ophthalmol 93:753-761

18. Matlach J, Hipp M, Wagner M, Heuschmann PU, Klink T, Grehn F (2015) A comparative study of a modified filtering trabeculotomy and conventional trabeculectomy. Clin Ophthalmol 9:483-492

19. Migdal C, Gregory W, Hitchings R (1994) Long-term functional outcome after early surgery compared with laser and medicine in open-angle glaucoma. Ophthalmology 101:1651-1656 (discussion 1657)

20. Musch DC, Brenda W, Gillespie BW, Lichter PR, Niziol LM, Janz NK (2009) Visual field progression in the Collaborative Initial Glaucoma Treatment Study-the impact of treatment and other baseline factors. Ophthalmology 116:200-207

21. Pfeiffer N, Grehn F (1992) Improved suture for fornix-based conjunctival flap in filtering surgery. Int Ophthalmol 16:391-396

22. Quigley HA, Broman AT (2006) The number of people with glaucoma worldwide in 2010 and 2020. Br J Ophthalmol 90:262-267

23. Riva I, Brusini P, Oddone F, Michelessi M, Weinreb RN, Quaranta L (2019) Canaloplasty in the treatment of open-angle glaucoma: a review of patient selection and outcomes. Adv Ther 36:31-43

24. Rulli E, Biagioli E, Riva I, Gambirasio G, De Simone I, Floriani I, Quaranta L (2013) Efficacy and safety of trabeculectomy vs nonpenetrating surgical procedures: a systematic review and metaanalysis. JAMA Ophthalmol 131:1573-1582

25. Roy S, Mermoud A (2017) Deep sclerectomy. Dev Ophthalmol 59:36-42

26. Sadruddin O, Pinchuk L, Angeles R, Palmberg $P$ (2019) Ab externo implantation of the MicroShunt a poly (styrene-block-isobutylene-block-styrene) surgical device for the treatment of primary openangle glaucoma: a review. Eye Vis (Lond) 6:36

27. Samuelson TW, Chang DF, Marquis R, Flowers B, Lim KS, Ahmed IIK, Jampel HD, Aung T, Crandall AS, Singh K (2019) A Schlemm canal microstent for intraocular pressure reduction in primary openangle glaucoma and cataract: the HORIZON study. Ophthalmology 126:29-37

28. Schlenker MB, Gulamhusein $\mathrm{H}$, Conrad-Hengerer I, Somers A, Lenzhofer M, Stalmans I, Reitsamer $\mathrm{H}$, Hengerer FH, Ahmed IIK (2017) Efficacy, safety, and risk factors for failure of standalone ab interno gelatin microstent implantation versus standalone trabeculectomy. Ophthalmology 124:1579-1588

29. Shaarawy TM, Sherwood MB, Grehn F (2009) WGA guidelines on design and reporting of glaucoma surgical trials. Kugler, Amsterdam

30. Sherwood MB, Migdal CS, Hitchings RA, Sharir M, Zimmerman TJ, Schultz JS (1993) Initial treatment of glaucoma: surgery or medications. Surv Ophthalmol 37:293-305

31. Tham YC, Li X, Wong TY, Quigley HA, Aung T, Cheng CY (2014) Global prevalence of glaucoma and projections of glaucoma burden through 2040: a systematic review and meta-analysis. Ophthalmology 121:2081-2090

32. Deutsche Ophthalmologische Gesellschaft e.V. (2012) Weißbuch zur Situation der ophthalmologischen Versorgung in Deutschland. https://www. dog.org/?cat=229. Zugegriffen: 10 Dez 2020.

33. World Health Organization (2016) Prevention of blindness and visual impairment. WHO library cataloguing-in-publication data 